\title{
Rare Ocular Complication in a Patient with Rabson-Mendenhall Syndrome
}

\author{
Karthik Kumar $^{1} \cdot$ Piyush Kohli $^{1}$ (D) Gautam Kumar ${ }^{1}$
}

Received: 8 April 2020 / Accepted: 22 June 2020 / Published online: 4 July 2020

(C) Dr. K C Chaudhuri Foundation 2020

To the Editor: Rabson-Mendenhall syndrome (RMS) is a rare disease characterized by severe insulin resistance caused by mutation in the insulin-receptor gene [1-3]. Being an extremely uncommon disease, ocular complications have never been reported earlier.

An 18-year-old female presented with sudden-onset visual loss in her left eye. She was a known case of RMS, diagnosed at the age of 9 year. She had a short stature, velvety hyperpigmentation of skin over nape of neck (acanthosis nigricans), generalized hirsutism, coarse facial features, broad nose, prognathism, anterior open bite; and irregular and crowded teeth.

Her best-corrected visual acuity was 20/20 in right eye and perception of light in left eye. Intraocular pressure (IOP) in her right eye was normal, and raised in left eye $(38 \mathrm{mmHg})$. Right eye examination was normal, while left eye had neovascularization of iris with vitreous hemorrhage (VH). B-scan ultrasonography showed VH with tractional retinal detachment. Complete blood count, peripheral smear, erythrocyte sedimentation rate, chest X-ray, Mantoux test, carotid doppler and echocardiography were normal. Hypercoagulable profile including factor $\mathrm{V}$ Leiden mutation; protein $\mathrm{C}$, protein $\mathrm{S}$ and antithrombin III deficiencies; homocysteine levels; and antiphospholipid antibodies was also normal. Human immunodeficiency virus and syphilis serologies were negative. She was diagnosed as neovascular glaucoma (NVG) secondary to probably central retinal vein occlusion (CRVO) in left eye, while right eye did not have diabetic changes.

She was advised IOP lowering medications followed by an urgent intravitreal anti-vascular epithelial growth factor

Piyush Kohli

kohli119@gmail.com

1 Department of Vitreo-retinal Services, Aravind Eye Hospital and Post Graduate Institute of Ophthalmology, Madurai, Tamil Nadu, India
(VEGF) injection and IOP lowering surgery. However, since her blood sugar levels were uncontrollable, she could not undergo any intraocular procedure and lost complete vision.

NVG is a potentially blinding disease caused by an excessive ocular VEGF load. Its treatment is immediate reduction of the VEGF load by either retinal laser and intravitreal antiVEGF injection as well as an immediate IOP control [4]. However, our patient could not undergo any ocular intervention as any surgical intervention with uncontrolled sugar levels predisposes to endophthalmitis i.e., intraocular infection [5].

As there is no specific treatment available for the management of RMS, therapy is generally supportive and in most cases all therapies prove unsuccessful [1-3]. The ocular complications related to the disease can be challenging to manage due to uncontrollable sugar levels.

\section{Compliance with Ethical Standards}

Conflict of Interest None.

\section{References}

1. Hassan I, Altaf H, Yaseen A. Rabson-Mendenhall syndrome. Indian J Dermatol. 2014;59:633.

2. Gupta J, Daniel JM, Vasudevan V. Rabson-Mendenhall syndrome. J Indian Soc Pedod Prev Dent. 2012;30:279-82.

3. American Diabetes Association. Diagnosis and classification of diabetes mellitus. Diabetes Care. 2013;36:S67-74.

4. Rodrigues GB, Abe RY, Zangalli C, et al. Neovascular glaucoma: A review. Int J Retina Vitreous. 2016;2:26.

5. Jabbarvand M, Hashemian H, Khodaparast M, Jouhari M, Tabatabaei A, Rezaei S. Endophthalmitis occurring after cataract surgery: outcomes of more than 480000 cataract surgeries, epidemiologic features, and risk factors. Ophthalmology. 2016;123:295-301.

Publisher's Note Springer Nature remains neutral with regard to jurisdictional claims in published maps and institutional affiliations. 